\title{
Cochrane
}

Library

Cochrane Database of Systematic Reviews

\section{Melatonin treatment for newborns with hypoxic ischaemic encephalopathy (Protocol)}

Hurley T, O'Dea M, Aslam S, Aly H, Robertson N, Molloy E

Hurley T, O'Dea M, Aslam S, Aly H, Robertson N, Molloy E.

Melatonin treatment for newborns with hypoxic ischaemic encephalopathy (Protocol).

Cochrane Database of Systematic Reviews 2020, Issue 10. Art. No.: CD013754.

DOI: 10.1002/14651858.CD013754.

www.cochranelibrary.com 
TABLE OF CONTENTS

HEADER 1

ABSTRACT

BACKGROUND

OBJECTIVES

METHODS

ACKNOWLEDGEMENTS

REFERENCES

APPENDICES

HISTORY

CONTRIBUTIONS OF AUTHORS

DECLARATIONS OF INTEREST

SOURCES OF SUPPORT 
[Intervention Protocol]

\section{Melatonin treatment for newborns with hypoxic ischaemic encephalopathy}

Tim Hurley¹, Mary O'Dea², Saima Aslam³ , Hany Aly4, Nikki Robertson5, Eleanor Molloy2,6

1Department of Paediatrics, Trinity College Dublin, Dublin, Ireland. 2Paediatric and Child Health, Trinity College Dublin, Dublin, Ireland. ${ }^{3}$ Neonatology, National Maternity Hospital, Dublin, Ireland. ${ }^{4}$ Neonatology, Cleveland Clinic Children's Hospital, Cleveland, OH, USA. 5Obstetrics and Gynaecology, University College London, London, UK. ${ }^{6}$ Department of Paediatrics, The National Children's Hospital, Tallaght, Dublin, Ireland

Contact address: Tim Hurley, hurleyti@tcd.ie.

Editorial group: Cochrane Neonatal Group.

Publication status and date: New, published in Issue 10, 2020.

Citation: Hurley T, O'Dea M, Aslam S, Aly H, Robertson N, Molloy E. Melatonin treatment for newborns with hypoxic ischaemic encephalopathy (Protocol). Cochrane Database of Systematic Reviews 2020, Issue 10. Art. No.: CD013754. DOI: 10.1002/14651858.CD013754.

Copyright ( 2020 The Cochrane Collaboration. Published by John Wiley \& Sons, Ltd.

\section{A B S T R A C T}

\section{Objectives}

This is a protocol for a Cochrane Review (intervention). The objectives are as follows:

To assess the effects and safety of melatonin compared to standard care, including therapeutic hypothermia, for improving survival and reducing neurological sequelae in newborns with neonatal encephalopathy. 


\section{B A C K G R O U N D}

\section{Description of the condition}

Neonatal encephalopathy (NE) is a clinically defined syndrome of disturbed neurological function in the earliest days of life in term and near-term newborns. Symptoms include an altered level of consciousness, seizures, poor tone, and an inability to initiate or maintain respiration (Volpe 2012). NE secondary to intrapartum events is estimated to affect 1.16 million babies per year, with the highest rates occurring in sub-Saharan Africa (Lee 2013). The estimated incidence of NE is one to three per 1000 live births in high-income settings, and up to 10 times higher in low- and middleincome settings (Kurinczuk 2010). NE is associated with multiorgan dysfunction (Shah 2004), disability, and a high incidence of early mortality. The term NE does not specify aetiology or pathogenesis (Molloy 2018), however there are many potential causes including hypoxic-ischaemic encephalopathy (HIE) and infectious, metabolic, coagulopathic, and placental causes. In up to half of cases, no underlying cause is identified (Nelson 2012). Regardless of the cause of NE, a final common pathway of hypoxiaischaemia leads to a cascade of events including oxidative stress (Zhao 2016) and systemic inflammation (Hagberg 2015) that finally result in cell death.

In the last decade, multiple clinical trials have demonstrated the benefit of therapeutic hypothermia (TH) for newborns with moderate to severe $\mathrm{NE}$, in settings with access to neonatal intensive care. There is clear evidence that $\mathrm{TH}$ reduces adverse outcomes at 18 months of age (Jacobs 2013); this improvement persists into childhood and there is widespread benefit to families, society, and the economy (Azzopardi 2014). For these reasons, TH is standard care for newborns with NE in high-income settings. In recent years, the search for adjunct interventions to $\mathrm{TH}$ has focused on other anti-inflammatory and anti-oxidant therapies, including allopurinol, erythropoietin, and magnesium sulphate. Although trials are ongoing, there is currently insufficient evidence to suggest that any of these therapies have a significant effect on outcomes in NE (Chaudhari 2012; Galinsky 2014; Robertson 2013; Wu 2015).

Despite treatment with $\mathrm{TH}$, almost half of those with moderate to severe NE die or survive with adverse neurodevelopmental outcomes (Azzopardi 2009). In addition, recent data suggest that newborns with mild NE (which is not routinely treated with $\mathrm{TH}$ ) are also at significant risk of neurodevelopmental disability (Chalak 2018). It is unclear whether TH is the best therapy for mild NE (Kariholu 2018), and there is a critical need for new therapies either single or adjunct - in NE generally.

\section{Description of the intervention}

Melatonin (N-acetyl-5-methoxytryptamine) is a naturally occurring hormone which is primarily released by the pineal gland in the brain (Epstein 1997). The release of melatonin is regulated by sensors in the eye which detect changes in light exposure. There is significant 24-hour variation in blood melatonin levels, with concentrations being lower in the day and higher at night. Melatonin is an important regulator of the circadian rhythm, but it also has antiinflammatory (Tarocco 2019), anti-oxidant (Reiter 2016), and antiapoptotic (Wang 2009) properties which make it a promising intervention for neuroprotection in NE.
Melatonin regulates the immune system in a number of ways. It acts as an anti-inflammatory molecule under conditions of exacerbated immune responses (Carrillo-Vico 2013). It exerts its influence on the innate immune system by regulating the lifespan of leukocytes by interfering with processes of cell death, regulating the release of pro-inflammatory cytokines and leukotrienes, and modulating the production of pro-inflammatory enzymes (Radogna 2010).

Melatonin is also a potent anti-oxidant. It indirectly stimulates the production of anti-oxidant enzymes including superoxide dismutase, glutathione peroxidase, and glutathione reductase, and inhibits the production of the pro-oxidant enzyme nitric oxide synthase (Reiter 2016). Melatonin is a direct free radical scavenger, reducing the toxic effects of reactive oxygen species and leading to further reduction in oxidative stress (Arteaga 2017). Melatonin reacts with reactive oxygen species to form N1-acetylN2-formyl-5-methoxykynuramine (AFMK). AFMK has potent antioxidant effects and scavenges radical oxygen species as the final melatonin compound involved in the free radical scavenging cascade (Claustrat 2015).

Melatonin exerts it effect via several different pathways. It readily crosses the blood-brain barrier and high levels accumulate in the central nervous system (Tan 2010). Because neurons have a high oxygen requirement, they are very vulnerable to oxidative stress. Some effects occur indirectly via two $G$ protein-coupled receptors, $M 1$ and $M 2$, which are widely distributed throughout the body and the brain (Stankov 1991), but melatonin also works through its diverse anti-oxidative mechanisms which prevent free-radical-induced oxidative damage to the electron transport chain and mitochondrial DNA (Reiter 2016). Melatonin is also believed to have a promising neuroprotective role through reduction of neuroinflammation (Esposito 2010); and in models of endotoxaemia it has been found to regulate the pro- and antiinflammatory cytokine network, modulate gene expression, and preserve mitochondrial integrity (Li 2013).

Several exogenous forms of ligands that attach to melatonin receptors are available and effective (Dubocovich 2010). However, very high pharmacological doses of melatonin may be required to exert anti-oxidant activity. In the piglet model, the beneficial effects of melatonin have been found to be dose-dependent, with earlier achievement of therapeutic levels (15 mg/L to $30 \mathrm{mg} / \mathrm{L}$ ) as important (Robertson 2019). Melatonin can be administered by the enteral or intravenous route, although the recommended route of administration for acute neuroprotection in the newborn is intravenous as it results in greater bioavailability (Balduini 2019). Supplementation with melatonin has demonstrated a rapid and sustained rise in plasma melatonin concentration in those undergoing TH (Balduini 2019). Melatonin is primarily metabolised in the liver via cytochrome CYP1A2 enzyme; this enzyme is involved in the metabolism of a number of other commonly used medications such as paracetamol and caffeine, which may affect the bioavailability of melatonin (Papagiannidou 2014). Melatonin has been studied in a number of conditions in the neonatal population and has an excellent safety profile (Gitto 2009).

\section{How the intervention might work}

Brain injury in NE results from the consequences of HIE, which include systemic inflammation, oxidative stress, and increased apoptotic activity (Zhao 2016) and ultimately result in cell death. Recent studies have shown that increased neutrophil activation 
(O'Hare 2015) and elevated circulating pro-inflammatory cytokines (O'Hare 2017) are associated with worse outcomes in NE. Clinical trials have demonstrated a reduction in circulating leukocytes and pro-inflammatory chemokines in patients treated with $\mathrm{TH}$ (Jenkins 2013), suggesting that modulation of brain injury in NE occurs via immunosuppression. Further studies have demonstrated that administration of melatonin leads to a downregulation of neutrophil activity and pro-inflammatory cytokines in the neonatal population (El-Gendy 2017; Gitto 2004; Gitto 2005).

Newborns are more vulnerable to oxidative stress than older individuals (Perrone 2013), and oxidative stress is a critical contributor to brain injury in NE. Newborns with NE are unable to compensate for the excess production of reactive oxygen species following hypoxic or ischaemic insult. This in turn leads to an accumulation of toxins and further systemic inflammation which results in worsening brain injury. Studies of several conditions involving oxidative stress in newborns, including perinatal asphyxia, sepsis, and respiratory distress syndrome, have demonstrated a reduction in oxidative stress with melatonin supplementation (Gitto 2009).

In $\mathrm{NE}$, oxidative stress combines with mitochondrial dysfunction to result in mitochondrial energy failure, a key regulator of apoptotic cell death (Lu 2015). Melatonin protects against neuron apoptosis via a wide range of effects, including reduced release of cytochrome c, increased expression of anti-apoptotic proteins, and reduced expression of pro-apoptotic proteins. These actions help prevent excess mitochondrial permeability and stabilise the mitochondrial membrane potential (Alonso-Alconada 2013), leading to reduced apoptotic cell death.

Several animal models of NE have demonstrated improved outcomes with melatonin supplementation. Neonatal rats with HIE brain injuries were at lower risk of learning deficits and behavioural asymmetry when treated with melatonin (Carloni 2008). Newborn mice with white matter brain lesions demonstrated improved secondary repair of these lesions when treated with melatonin (Husson 2002). A piglet model of HIE demonstrated improved neuroprotection as measured by magnetic resonance spectroscopy when melatonin treatment was combined with TH, compared to TH alone (Robertson 2013). Lamb and rat models of NE have demonstrated that the neuroprotective effects of melatonin treatment are mediated through reduced proinflammatory markers, reduced apoptosis-mediated cell death and reduced cerebral oxidative stress (Aridas 2018; Villapol 2011).

Melatonin has an excellent safety profile: it is well tolerated both in high doses by adults, with no adverse effects on sedation (Andersen 2016), and by children on long-term treatment, with no suppression of endogenous melatonin secretion (Palm 1997). It has been studied in many conditions in the neonatal population, including respiratory distress syndrome (Gitto 2004) and neonatal sepsis (Gitto 2001), with no adverse effects reported. The largest safety study of melatonin in the neonatal population, a retrospective study of 85 neonatal patients enrolled in multiple clinical trials of different conditions, demonstrated improved clinical outcomes and no adverse events (Aversa 2012). Studies have not demonstrated any evidence of toxicity, even when administered at very high doses (Aversa 2012). Enteral and intravenous administration of melatonin are feasible and have demonstrated increased plasma concentration of melatonin. It has also been shown following melatonin administration, neonates have reduced clearance and prolonged half-life compared to adults. Hypothermia does not appear to cause any significant changes in pharmacokinetics in the neonatal population (Balduini 2019), therefore melatonin could make a good adjunct to HT.

\section{Why it is important to do this review}

Melatonin is not currently in routine use for infants with NE. There are compelling data from animal studies and early clinical trials to suggest melatonin is safe and may be effective as a neuroprotective agent in infants with NE, either as an adjunct or single therapy. Our aim is to perform a systematic review of clinical studies on the use of melatonin for babies with NE.

\section{O B JECT IVES}

To assess the effects and safety of melatonin compared to standard care, including therapeutic hypothermia, for improving survival and reducing neurological sequelae in newborns with neonatal encephalopathy.

\section{METHODS}

\section{Criteria for considering studies for this review}

\section{Types of studies}

We will include all randomised and quasi-randomised controlled trials comparing the use of melatonin with standard care for patients with neonatal encephalopathy.

\section{Types of participants}

We will include infants born at or beyond 35 weeks' gestation, with no major congenital malformations at birth and meeting any of the following criteria.

1. Evidence of perinatal asphyxia, with each enrolled infant satisfying at least one of the following criteria

a. Apgar score of five or less at 10 minutes

b. Mechanical ventilation or resuscitation at 10 minutes

c. Cord pH of less than 7.1, or an arterial pH of less than 7.1 or base deficit of 12 or more within 60 minutes of birth

2. Evidence of encephalopathy according to Sarnat staging (Sarnat 1976)

a. Stage 1 (mild): hyperalertness, hyperreflexia, dilated pupils, tachycardia, absence of seizures

b. Stage 2 (moderate): lethargy, hyperreflexia, miosis, bradycardia, seizures, hypotonia with weak suck and Moro

c. Stage 3 (severe): stupor, flaccidity, small to mid-position pupils that react poorly to light, decreased stretch reflexes, hypothermia and absent Moro reflex

3. Severity of encephalopathy according to Thompson Score (Thompson 1997)

a. Score 0 to 10 (mild)

b. Score 11 to 14 (moderate)

c. Score 15 or more (severe)

\section{Types of interventions}

The intervention will be melatonin supplementation, by oral or intravenous administration, given to patients with NE within the first week of life. The comparison will be with standard treatment for NE, including TH. 


\section{Types of outcome measures}

\section{Primary outcomes}

1. Composite of death or long-term major neurodevelopmental disability, defined as a diagnosis of cerebral palsy (CP); Gross Motor Function Classification System (GMFCS) score of three or more; developmental delay, defined as a score on any standardised infant developmental assessment of more than two standard deviations below the mean; intellectual disability, defined as intelligence quotient (IQ) of more than two standard deviations below the mean; visual impairment, defined as visual acuity of less than $6 / 60$ in both eyes; sensorineural hearing loss, defined as unable to hear sounds less than 40 decibels; or the need for more than one anticonvulsant medication for seizure control. Long-term outcomes will be reported for all studies that have evaluated children after 18 months of age.

\section{Secondary outcomes}

1. Mortality

a. Early (within the first week of life)

b. Late (within the first year of life)

c. At 18 months or final follow-up assessment

2. Each component of major neurodevelopmental disability assessed at more than 18 months of age

a. Cerebral palsy (Richards 2013)

b. Gross Motor Function Classification System of three or more (McDowell 2008)

c. Developmental delay (Johnson 2014): defined as a score on any standardised infant developmental assessment of more than two standard deviations below the mean

d. Intellectual disability (Schalock 2007): defined as IQ of more than two standard deviations below the mean

e. Visual impairment (Dandona 2006): defined as visual acuity of less than $6 / 60$ in both eyes

f. Sensorineural hearing impairment (Morzaria 2004): defined as unable to hear sounds less than 40 decibels

3. Abnormal magnetic resonance imaging (Barkovich 1998)

4. Multiorgan dysfunction (Shah 2004)

5. Use of anti-convulsant medications

\section{Search methods for identification of studies}

We will use the criteria and standard methods of Cochrane and Cochrane Neonatal (see the Cochrane Neonatal standard search strategy).

\section{Electronic searches}

We will conduct a comprehensive search of databases including: the Cochrane Central Register of Controlled Trials (CENTRAL 2020 current issue) in the Cochrane Library; Ovid MEDLINE Epub Ahead of Print, In-Process \& Other Non-Indexed Citations, Ovid MEDLINE Daily and Ovid MEDLINE (1946 to current); and CINAHL (1981 to current). We will not apply language restrictions to the searches. We include the MEDLINE search in Appendix 1, which will be translated into the other databases using the standard search strategy of Cochrane Neonatal (Appendix 2).

We will search clinical trial registries for ongoing or recently completed trials. We will search The World Health Organization's International Clinical Trials Registry Platform
(ICTRP) (www.who.int/ictrp/search/en/) and the National Library of Medicine's ClinicalTrials.gov (clinicaltrials.gov) via CENTRAL. Additionally, we will search the ISRCTN Registry for any unique trials not found through the CENTRAL search.

\section{Searching other resources}

We will search the reference lists of previous reviews, and trials included in the current review, for citations and cross-references. We will search abstracts from conference proceedings, including those of the joint European Neonatal Societies/European Society for Paediatric Research (jENS/ESPR) (1960 to present), the Pediatric Academic Societies (PAS) (1998 to present) and the Perinatal Society of Australia and New Zealand (PSANZ) (1998 to present). We will also consult experts in the subject area.

\section{Data collection and analysis}

We will collect and analyse data in accordance with the standard methods of Cochrane Neonatal.

\section{Selection of studies}

Two review authors will independently assess the eligibility of all retrieved studies for inclusion in the review. Disagreements will be resolved by discussion between the two review authors. We will assess studies in the usual consecutive format, starting with title and abstract screening and subsequently full-text screening. We will use Covidence software to facilitate this process (Covidence 2019). We will document the study selection process in a PRISMA flow diagram.

\section{Data extraction and management}

Two review authors will independently perform data extraction using a structured form. Differences will be resolved by discussion between the two review authors. We will contact study authors to request additional data from unpublished studies in abstract form, if required. All analyses will be performed using Review Manager 5 software (Review Manager 2014).

\section{Assessment of risk of bias in included studies}

Two review authors (TH and MOD) will independently assess all included trials using the Cochrane 'Risk of bias' tool. Each study will be assigned a judgement of low, high, or unclear risk of bias for the following domains (Higgins 2019).

1. Sequence generation (selection bias)

2. Allocation concealment (selection bias)

3. Blinding of participants and personnel (performance bias)

4. Blinding of outcome assessment (detection bias)

5. Incomplete outcome data (attrition bias)

6. Selective reporting (reporting bias)

7. Any other bias

Any disagreements will be resolved by discussion or by consulting a third review author. See Appendix 3 for a more detailed description of risk of bias for each domain.

\section{Measures of treatment effect}

We will express treatment effects for dichotomous outcomes using summary risk ratio (RR), risk difference (RD), number needed to treat for an additional beneficial outcome (NNTB) or number 
needed to treat for an additional harmful outcome (NNTH), with $95 \%$ confidence intervals (Cls). For continuous outcomes, we will express treatment effects using mean difference (MD) and 95\% $\mathrm{Cls}$, where outcomes were measured using the same scale or in the same way in the included studies. We will used standardised mean difference (SMD) where studies assess the same outcome but measure it in different ways.

\section{Unit of analysis issues}

Our primary outcome is a composite of dichotomous outcomes. Each constituent of the primary outcome is clearly defined by standardised criteria and expressed in standardised unit measurement. Where outcomes are expressed as continuous data, we will use internationally accepted cut-off values to categorise participants, as outlined above.

For cluster-randomised trials we will follow the guidance in the Cochrane Handbook for Systematic Reviews of Interventions (Higgins 2019) in order to avoid unit of analysis errors. We will conduct the analysis at the same level as the allocation, using a summary measurement from each cluster, so each cluster will become the individual and will be analysed on this basis. Cross-over trials will be excluded.

\section{Dealing with missing data}

Our strategy to deal with missing data will follow Cochrane Handbook (Higgins 2019) guidance, as follows.

1. Where possible, we will contact original investigators for missing data.

2. Where possible, missing standard deviations will be imputed using the coefficient of variation (CV), or calculated from other available statistics including standard errors, $\mathrm{Cls}$, $\mathrm{t}$ values, and $\mathrm{P}$ values.

3. If the data are assumed to be missing at random, they will be analysed without imputing any missing values.

4. If data cannot be assumed to be missing at random then the missing outcomes will be imputed with replacement values, assuming all to have a poor outcome.

5. If we are required to impute data, we will make explicit the assumptions of any methods used.

6. We will perform sensitivity analyses to assess how sensitive results are to reasonable changes in the assumptions that are made.

7. We will address the potential impact of missing data on the findings of the review in the 'Discussion' section.

\section{Assessment of heterogeneity}

We will estimate the treatment effects of individual trials and examine heterogeneity among trials by inspecting the forest plots and quantifying the impact of heterogeneity using the $\mathrm{I}^{2}$ statistic. We will grade the degree of heterogeneity as: less than $25 \%$ no heterogeneity; $25 \%$ to $49 \%$ low heterogeneity; $50 \%$ to $75 \%$ moderate heterogeneity; more than $75 \%$ substantial heterogeneity. If we note statistical heterogeneity $\left(\mathrm{I}^{2}>50 \%\right)$, we will explore the possible causes (e.g. differences in study quality, participants, intervention regimens, or outcome assessments).

\section{Assessment of reporting biases}

We will assess publication bias by creating funnel plots for metaanalyses consisting of at least 10 studies. If significant asymmetry is found on visual inspection of the funnel plots, we will report this in the corresponding results.

\section{Data synthesis}

We will perform a meta-analysis of primary and secondary outcomes, as well as pre-defined subgroups, subject to availability of data. We will use standardised methodologies as described in the Cochrane Handbook (Higgins 2019). Meta-analysis will be conducted using the inverse variance method for continuous outcomes, and the Mantel-Haenzel method for dichotomous outcomes. We will use the random-effects model and present all our results with $95 \%$ Cls. We will calculate the RR, RD, and NNTB or NNTH if the RD is significant - each with $95 \% \mathrm{Cls}$ - for categorical outcomes. We will calculate the MD with $95 \% \mathrm{Cls}$ for continuous outcomes. Where continuous outcomes are measured using different scales, the treatment effect will be expressed as SMD with $95 \% \mathrm{Cls}$. For any outcomes where the included studies are not sufficiently homogeneous, or where insufficient data are available for meta-analysis, we will present a narrative synthesis.

\section{Certainty of evidence}

We will use the GRADE approach, as outlined in the GRADE Handbook (Schünemann 2013), to assess the certainty of evidence for the following (clinically relevant) outcomes: mortality, presence of cerebral palsy, developmental delay or intellectual disability, visual impairment or sensorineural hearing loss, abnormal magnetic resonance imaging, multiorgan dysfunction, and use of anti-convulsant medications.

Two review authors will independently assess the certainty of the evidence for each of the outcomes above. We will consider evidence from randomised controlled trials as high-certainty but will downgrade the evidence by one level for serious (or two levels for very serious) limitations based upon the following: design (risk of bias), consistency across studies, directness of the evidence, precision of estimates, and presence of publication bias. We will use GRADEpro GDT (GRADEpro GDT) to create a 'Summary of findings' table to report the certainty of the evidence.

The GRADE approach results in an assessment of the certainty of a body of evidence, of one of the following four grades.

1. High certainty: we are very confident that the true effect lies close to that of the estimate of the effect.

2. Moderate certainty: we are moderately confident in the effect estimate; the true effect is likely to be close to the estimate of the effect, but there is a possibility that it is substantially different.

3. Low certainty: our confidence in the effect estimate is limited; the true effect may be substantially different from the estimate of the effect.

4. Very low certainty: we have very little confidence in the effect estimate; the true effect is likely to be substantially different from the estimate of effect.

\section{Subgroup analysis and investigation of heterogeneity}

Where there are sufficient studies, the following a priori subgroup analyses will be conducted. 
1. Severity of NE

2. Timing of initiation of melatonin treatment

3. Method of melatonin administration

4. Dose of melatonin administration $(0.5 \mathrm{mg} / \mathrm{kg} /$ day to $3.0 \mathrm{mg} / \mathrm{kg} /$ day; $3.1 \mathrm{mg} / \mathrm{kg} /$ day to $7.5 \mathrm{mg} / \mathrm{kg} /$ day; $7.6 \mathrm{mg} / \mathrm{kg} /$ day to $20 \mathrm{mg} /$ $\mathrm{kg} /$ day)

5. Duration of melatonin administration (single dose; one to three days; four to seven days; more than seven days)

6. Daily timing of melatonin administration (day (8am to $8 \mathrm{pm}$ ); night (8pm to 8am))

7. Adjunct use of $\mathrm{TH}$

\section{Sensitivity analysis}

Where we identify substantial heterogeneity, we will conduct sensitivity analysis to determine if the findings are affected by inclusion of only those trials considered to have used adequate methodology with a low risk of bias (selection and performance bias). We will report results of sensitivity analyses for primary outcomes only.

\section{ACK N O WLEDGEMENTS}

The methods section of this protocol is based on a standard template used by Cochrane Neonatal.

We would like to thank Cochrane Neonatal: Colleen Ovelman (Managing Editor), Caitlin O'Connell Eckert (Assistant Managing Editor), Roger Soll (Co-coordinating editor), and Bill McGuire (Cocoordinating Editor), who provided editorial and administrative support. Carol Friesen, Cochrane Neonatal Information Specialist, developed the Ovid MEDLINE search strategy, and Colleen Ovelman peer-reviewed the strategy. Marie Berg and Jacqueline Ho peerreviewed the protocol. 


\section{REFERE N C E S}

\section{Additional references}

\section{Alonso-Alconada 2013}

Alonso-Alconada D, Álvarez A, Arteaga O, Martínez-Ibargüen A, Hilario E. Neuroprotective effect of melatonin: a novel therapy against perinatal hypoxia-ischemia. International Journal of Molecular Sciences 2013;14(5):9379-95. [DOI: 10.3390/ ijms14059379] [PMID: 23629670]

\section{Andersen 2016}

Andersen LP, Werner MU, Rosenkilde MM, Fenger AQ, Petersen MC, Rosenberg J, et al. Pharmacokinetics of highdose intravenous melatonin in humans. Journal of Clinical Pharmacology 2016;56(3):324-9. [DOI: 10.1002/jcph.592] [PMID: 26184078]

\section{Aridas 2018}

Aridas JDS, Yawno T, Sutherland AE, Nitsos I, Ditchfield M, Wong FY, et al. Systemic and transdermal melatonin administration prevents neuropathology in response to perinatal asphyxia in newborn lambs. Journal of Pineal Research 2018;64(4):e12479. [PMID: 29464766]

\section{Arteaga 2017}

Arteaga O, Álvarez A, Revuelta M, Santaolalla F, Urtasun A, Hilario E. Role of antioxidants in neonatal hypoxic-ischemic brain injury: new therapeutic approaches. International Journal of Molecular Sciences 2017;18(2):265. [DOI: 10.3390/ ijms18020265] [PMID: 28134843]

\section{Aversa 2012}

Aversa S, Marseglia L, Arco A, D’Angelo G, Cusumano E, Barberi I, et al. 1640 efficacy and safety of melatonin in neonates. Archives Of Disease in Childhood 2012;97 Suppl 2:A464.

\section{Azzopardi 2009}

Azzopardi DV, Strohm B, Edwards AD, Dyet L, Halliday HL, Juszczak E, et al. Moderate hypothermia to treat perinatal asphyxial encephalopathy. New England Journal of Medicine 2009;361(14):1349-58. [DOI: 10.1056/NEJMoa0900854] [PMID: $19797281]$

\section{Azzopardi 2014}

Azzopardi D, Strohm B, Marlow N, Brocklehurst P, Deierl A, Eddama O, Goodwin J, Halliday HL, Juszczak E, Kapellou O, Levene M. Effects of hypothermia for perinatal asphyxia on childhood outcomes. New England Journal of Medicine 2014;371(2):140-9.

\section{Balduini 2019}

Balduini W, Weiss MD, Carloni S, Rocchi M, Sura L, Rossignol C, et al. Melatonin pharmacokinetics and dose extrapolation after enteral infusion in neonates subjected to hypothermia. Journal of Pineal Research 2019;66(4):e12565. [DOI: 10.1111/jpi.12565] [PMID: 30734962]

\section{Barkovich 1998}

Barkovich AJ, Hajnal BL, Vigneron D, Sola A, Partridge JC, Allen $\mathrm{F}$, et al. Prediction of neuromotor outcome in perinatal asphyxia: evaluation of MR scoring systems. American Journal of Neuroradiology 1998;19(1):143-9. [PMID: 9432172]

\section{Carloni 2008}

Carloni S, Perrone S, Buonocore G, Longini M, Proietti F, Balduini W. Melatonin protects from the long-term consequences of a neonatal hypoxic-ischemic brain injury in rats. Journal of Pineal Research 2008;44(2):157-64. [DOI: 10.1111/j.1600-079X.2007.00503.x] [PMID: 18289167]

\section{Carrillo-Vico 2013}

Carrillo-Vico A, Lardone PJ, Álvarez-Sánchez N, RodríguezRodríguez A, Guerrero JM. Melatonin: buffering the immune system. International Journal of Molecular Sciences 2013;14(4):8638-83. [DOI: 10.3390/ijms14048638] [PMID: 23609496]

\section{Chalak 2018}

Chalak LF, Nguyen KA, Prempunpong C, Heyne R, Thayyil S, Shankaran S, et al. Prospective research in infants with mild encephalopathy identified in the first six hours of life: neurodevelopmental outcomes at 18-22 months. Pediatric Research 2018;84(6):861. [DOI: 10.1038/s41390-018-0174-x] [PMID: 30250303]

\section{Chaudhari 2012}

Chaudhari T, McGuire W. Allopurinol for preventing mortality and morbidity in newborn infants with hypoxicischaemic encephalopathy. Cochrane Database of Systematic Reviews 2012, Issue 7. Art. No: CD006817. [DOI: 10.1002/14651858.CD006817.pub3] [PMID: 22786499]

\section{Claustrat 2015}

Claustrat B, Leston J. Melatonin: physiological effects in humans. Neurochirurgie 2015;61(2-3):77-84. [DOI: 10.1016/ j.neuchi.2015.03.002] [PMID: 25908646]

\section{Covidence 2019 [Computer program]}

Veritas Health Innovation Covidence. Melbourne, Australia: Veritas Health Innovation, 2019.

\section{Dandona 2006}

Dandona L, Dandona R. Revision of visual impairment definitions in the International Statistical Classification of Diseases. BMC Medicine 2006;4(1):7. [DOI: 10.1186/1741-7015-4-7] [PMID: 16539739]

\section{Dubocovich 2010}

Dubocovich ML, Delagrange P, Krause DN, Sugden D, Cardinali DP, Olcese J. International union of basic and clinical pharmacology. LXXV. Nomenclature, classification, and pharmacology of $\mathrm{G}$ protein-coupled melatonin receptors. Pharmacological Reviews 2010;62(3):343-80. [DOI: 10.1124/ pr.110.002832] [PMID: 20605968]

\section{El-Gendy 2017}

El-Gendy FM, El-Hawy MA, Hassan MG. Beneficial effect of melatonin in the treatment of neonatal sepsis. Journal of 
Maternal-Fetal \& Neonatal Medicine 2017;31(17):1-16. [DOI: 10.1080/14767058.2017.1342794] [PMID: 28612668]

\section{Epstein 1997}

Brzezinski A. Melatonin in humans. New England Journal of Medicine 1997;336(3):186-95. [DOI: 10.1056/ NEJM199701163360306] [PMID: 8988899]

\section{Esposito 2010}

Esposito E, Cuzzocrea S. Antiinflammatory activity of melatonin in central nervous system. Current Neuropharmacology 2010;8(3):228-42. [DOI: 10.2174/157015910792246155] [PMID: 21358973]

\section{Galinsky 2014}

Galinsky R, Bennet L, Groenendaal F, Lear CA, Tan S, van Bel F, et al. Magnesium is not consistently neuroprotective for perinatal hypoxia-ischemia in term-equivalent models in preclinical studies: a systematic review. Developmental Neuroscience 2014;36(2):73-82. [DOI: 10.1159/000362206] [PMID: 24854050]

\section{Gitto 2001}

Gitto E, Karbownik M, Reiter RJ, Tan DX, Cuzzocrea S, Chiurazzi P, et al. Effects of melatonin treatment in septic newborns. Pediatric Research 2001;50(6):756. [DOI: 10.1203/00006450-200112000-00021] [PMID: 11726736]

\section{Gitto 2004}

Gitto E, Reiter RJ, Cordaro SP, La Rosa M, Chiurazzi P, Trimarchi G, et al. Oxidative and inflammatory parameters in respiratory distress syndrome of preterm newborns: beneficial effects of melatonin. American Journal of Perinatology 2004;21(4):209-16. [DOI: 10.1055/s-2004-828610] [PMID: 15168319]

\section{Gitto 2005}

Gitto E, Reiter RJ, Sabatino G, Buonocore G, Romeo C, Gitto P, et al. Correlation among cytokines, bronchopulmonary dysplasia and modality of ventilation in preterm newborns: improvement with melatonin treatment. Journal of Pineal Research 2005;39(3):287-93. [DOI: 10.1111/j.1600-079X.2005.00251.x] [PMID: 16150110]

\section{Gitto 2009}

Gitto E, Pellegrino S, Gitto P, Barberi I, Reiter RJ. Oxidative stress of the newborn in the pre- and postnatal period and the clinical utility of melatonin. Journal of Pineal Research 2009;46(2):128-39. [DOI: 10.1111/j.1600-079X.2008.00649.x] [PMID: 19054296]

\section{GRADEpro GDT [Computer program]}

GRADEpro Guideline Development Tool [Software]. McMaster University, 2020 (developed by Evidence Prime, Inc).

\section{Hagberg 2015}

Hagberg H, Mallard C, Ferriero DM, Vannucci SJ, Levison SW, Vexler ZS, et al. The role of inflammation in perinatal brain injury. Nature Reviews Neurology 2015;11(4):192-208. [DOI: 10.1038/nrneurol.2015.13] [PMID: 25686754]

\section{Higgins 2011}

Higgins JP, Green S, editor(s). Cochrane Handbook for Systematic Reviews of Interventions Version 5.1.0 (updated March 2011). The Cochrane Collaboration, 2011. Available from handbook.cochrane.org.

\section{Higgins 2019}

Higgins JPT, Thomas J, Chandler J, Cumpston M, Li T, Page MJ, Welch VA (editors). Cochrane Handbook for Systematic Reviews of Interventions version 6.0 (updated July 2019). Cochrane, 2019. Available from www.training.cochrane.org/handbook.

\section{Husson 2002}

Husson I, Mesplès B, Bac P, Vamecq J, Evrard P, Gressens P. Melatoninergic neuroprotection of the murine periventricular white matter against neonatal excitotoxic challenge. Annals of Neurology 2002;51(1):82-92. [PMID: 11782987]

\section{Jacobs 2013}

Jacobs SE, Berg M, Hunt R, Tarnow-Mordi WO, Inder TE, Davis PG. Cooling for newborns with hypoxic ischaemic encephalopathy. Cochrane Database of Systematic Reviews 2013, Issue 1. Art. No: CD003311. [DOI: 10.1002/14651858.CD003311.pub3]

\section{Jenkins 2013}

Jenkins, DD, Lee T, Chiuzan C, Perkel JK, Rollins LG, Wagner CL, et al. Altered circulating leukocytes and their chemokines in a clinical trial of therapeutic hypothermia for neonatal hypoxic ischemic encephalopathy. Pediatric Critical Care Medicine 2013;14(8):786-95. [DOI: 10.1097/PCC.0b013e3182975cc9] [PMID: 23897243]

\section{Johnson 2014}

Johnson S, Moore T, Marlow N. Using the Bayley-III to assess neurodevelopmental delay: which cut-off should be used? Pediatric Research 2014;75(5):670. [DOI: 10.1038/pr.2014.10] [PMID: 24492622]

\section{Kariholu 2018}

Kariholu U, Montaldo P, Markati T, Lally PJ, Pryce R, Teiserskas J, et al. Therapeutic hypothermia for mild neonatal encephalopathy: a systematic review and meta-analysis. Archives of Disease in Childhood-Fetal and Neonatal Edition 2018;19. [DOI: 10.1136/archdischild-2018-315711] [PMID: 30567775]

\section{Kurinczuk 2010}

Kurinczuk JJ, White-Koning M, Badawi N. Epidemiology of neonatal encephalopathy and hypoxic-ischaemic encephalopathy. Early Human Development 2010;86(6):329-38. [DOI: 10.1016/j.earlhumdev.2010.05.010] [PMID: 20554402]

\section{Lee 2013}

Lee AC, Kozuki N, Blencowe H, Vos T, Bahalim A, Darmstadt GL, et al. Intrapartum-related neonatal encephalopathy incidence and impairment at regional and global levels for 2010 with trends from 1990. Pediatric Research 2013;74(S1):50. [DOI: 10.1038/pr.2013.206] [PMID: 24366463] 


\section{Li 2013}

Li CX, Liang DD, Xie GH, Cheng BL, Chen QX, Wu SJ, et al. Altered melatonin secretion and circadian gene expression with increased proinflammatory cytokine expression in early-stage sepsis patients. Molecular Medicine Reports 2013;7(4):1117-22. [DOI: 10.3892/mmr.2013.1331] [PMID: 23426900]

\section{Lu 2015}

Lu Y, Tucker D, Dong Y, Zhao N, Zhuo X, Zhang Q. Role of mitochondria in neonatal hypoxic-ischemic brain injury. Journal of Neuroscience and Rehabilitation 2015;2(1):1-14. [PMID: 27441209]

\section{McDowell 2008}

McDowell B. The gross motor function classification system - expanded and revised. Developmental Medicine \& Child Neurology 2008;50(10):725.

\section{Miller 2004}

Miller SP, Latal B, Clark H, Barnwell A, Glidden D, Barkovich AJ, et al. Clinical signs predict 30-month neurodevelopmental outcome after neonatal encephalopathy. American Journal of Obstetrics And Gynecology 2004;190(1):93-9. [DOI: 10.1016/ s0002-9378(03)00908-6] [PMID: 14749642]

\section{Molloy 2018}

Molloy EJ, Bearer C. Neonatal encephalopathy versus hypoxicischemic encephalopathy. Pediatric Research 2018;84(5):574. [DOI: 10.1038/s41390-018-0169-7] [PMID: 30214023]

\section{Morzaria 2004}

Morzaria S, Westerberg BD, Kozak FK. Systematic review of the etiology of bilateral sensorineural hearing loss in children. International Journal of Pediatric Otorhinolaryngology 2004;68(9):1193-8. [DOI: 10.1016/j.ijporl.2004.04.013] [PMID: 15302152]

\section{Nelson 2012}

Nelson KB, Bingham P, Edwards EM, Horbar JD, Kenny MJ, Inder T, et al. Antecedents of neonatal encephalopathy in the Vermont Oxford Network encephalopathy registry. Pediatrics 2012;130(5):878-86. [DOI: 10.1542/peds.2012-0714] [PMID: 23071210]

\section{O'Hare 2017}

O'Hare FM, Watson RW, O'Neill A, Segurado R, Sweetman D, Downey $P$, et al. Serial cytokine alterations and abnormal neuroimaging in newborn infants with encephalopathy. Acta Paediatrica 2017;106(4):561-7. [DOI: 10.1111/apa.13745] [PMID: 28097694]

\section{O'Hare 2015}

O'Hare FM, Watson RW, O'Neill A, Blanco A, Donoghue V, Molloy EJ. Persistent systemic monocyte and neutrophil activation in neonatal encephalopathy. Journal of Maternal-Fetal \& Neonatal Medicine 2015;29(4):582-9. [DOI: 10.3109/14767058.2015.1012060] [PMID: 25694256]

\section{Palm 1997}

Palm L, Blennow G, Wetterberg L. Long-term melatonin treatment in blind children and young adults with circadian sleep-wake disturbances. Developmental Medicine \& Child Neurology 1997;39(5):319-25. [DOI: 10.1111/ j.1469-8749.1997.tb07438.x] [PMID: 9236698]

\section{Papagiannidou 2014}

Papagiannidou E, Skene DJ, loannides C. Potential drug interactions with melatonin. Physiology \& Behavior 2014;131:17-24. [DOI: 10.1016/j.physbeh.2014.04.016] [PMID: 24732412]

\section{Perrone 2013}

Perrone S, Tataranno LM, Stazzoni G, Ramenghi L, Buonocore G. Brain susceptibility to oxidative stress in the perinatal period. Journal of Maternal-Fetal \& Neonatal Medicine 2013;28 Suppl 1:2291-5. [DOI: 10.3109/14767058.2013.796170] [PMID: 23968388]

\section{Radogna 2010}

Radogna F, Diederich M, Ghibelli L. Melatonin: a pleiotropic molecule regulating inflammation. Biochemical Pharmacology 2010;80(12):1844-52. [DOI: 10.1016/j.bcp.2010.07.041] [PMID: 20696138]

\section{Reiter 2016}

Reiter RJ, Mayo JC, Tan DX, Sainz RM, Alatorre-Jimenez M, Qin L. Melatonin as an antioxidant: under promises but over delivers. Journal of Pineal Research 2016;61(3):253-78. [DOI: 10.1111/ jpi.12360] [PMID: 27500468]

\section{Review Manager 2014 [Computer program]}

Nordic Cochrane Centre, The Cochrane Collaboration Review Manager 5 (RevMan 5). Version 5.3. Copenhagen: Nordic Cochrane Centre, The Cochrane Collaboration, 2014.

\section{Richards 2013}

Richards CL, Malouin F. Chapter 18: Cerebral palsy definition, assessment and rehabilitation. In: Handbook of Clinical Neurology. Elsevier, 2013:183-95.

\section{Robertson 2013}

Robertson NJ, Faulkner S, Fleiss B, Bainbridge A, Andorka $C$, Price $D$, et al. Melatonin augments hypothermic neuroprotection in a perinatal asphyxia model. Brain 2013;136(1):90-105. [DOI: 10.1093/brain/aws285] [PMID: 23183236]

\section{Robertson 2019}

Robertson NJ, Martinello K, Lingam I, Avdic-Belltheus A, Meehan C, Alonso-Alconada D, et al. Melatonin as an adjunct to therapeutic hypothermia in a piglet model of neonatal encephalopathy: a translational study. Neurobiology Of Disease 2019;121:240-51.

\section{Sarnat 1976}

Sarnat HB, Sarnat MS. Neonatal encephalopathy following fetal distress: a clinical and electroencephalographic study. Archives of Neurology 1976;33(10):696-705. [DOI: 10.1001/ archneur.1976.00500100030012] [PMID: 987769] 


\section{Schalock 2007}

Schalock RL, Luckasson RA, Shogren KA, BorthwickDuffy S, Bradley V, Buntinx WH, et al. The renaming of mental retardation: understanding the change to the term intellectual disability. Intellectual and Developmental Disabilities 2007;45(2):116-24. [DOI: 10.1352/1934-9556(2007)45[116:TROMRU]2.0.CO;2] [PMID: 17428134]

\section{Schünemann 2013}

Schünemann H, Brożek J, Guyatt G, Oxman A, editor(s). Handbook for grading the quality of evidence and the strength of recommendations using the GRADE approach (updated October 2013). GRADE Working Group, 2013. Available from gdt.guidelinedevelopment.org/app/handbook/handbook.html.

\section{Shah 2004}

Shah P, Riphagen S, Beyene J, Perlman M. Multiorgan dysfunction in infants with post-asphyxial hypoxic-ischaemic encephalopathy. Archives of Disease in Childhood. Fetal And Neonatal Edition 2004;89(2):F152-5. [DOI: 10.1136/ adc.2002.023093] [PMID: 14977901]

\section{Stankov 1991}

Stankov B, Fraschini F, Reiter RJ. Melatonin binding sites in the central nervous system. Brain Research Reviews 1991;16(3):245-56. [PMID: 1665096]

\section{Tan 2010}

Tan DX. Melatonin and brain. Current Neuropharmacology 2010;8(3):161. [DOI: 10.2174/157015910792246263] [PMID: 21358966]

\section{Tarocco 2019}

Tarocco A, Caroccia N, Morciano G, Wieckowski MR, Ancora G, Garani G, et al. Melatonin as a master regulator of cell death and inflammation: molecular mechanisms and clinical implications for newborn care. Cell Death \& Disease 2019;10(4):317. [DOI: 10.1038/s41419-019-1556-7] [PMID: 30962427]

\section{Thompson 1997}

Thompson CM, Puterman AS, Linley LL, Hann FM, Elst CW, Molteno CD, et al. The value of a scoring system for hypoxic ischaemic encephalopathy in predicting neurodevelopmental outcome. Acta Paediatrica 1997;86(7):757-61. [PMID: 9240886]

\section{Villapol 2011}

Villapol S, Fau S, Renolleau S, Biran V, Charriaut-Marlangue C, Baud O. Melatonin promotes myelination by decreasing white matter inflammation after neonatal stroke. Pediatric Research 2011;69(1):51-5. [PMID: 20856166]

\section{Volpe 2012}

Volpe JJ. Neonatal encephalopathy: an inadequate term for hypoxic-ischemic encephalopathy. Annals of Neurology 2012;72(2):156-66. [DOI: 10.1002/ana.23647] [PMID: 22926849]

\section{Wang 2009}

Wang X. The antiapoptotic activity of melatonin in neurodegenerative diseases. CNS Neuroscience \& Therapeutics 2009;15(4):345-57. [DOI: 10.1111/j.1755-5949.2009.00105.x] [PMID: 19818070]

\section{Wu 2015}

Wu YW, Gonzalez FF. Erythropoietin: a novel therapy for hypoxic-ischaemic encephalopathy? Developmental Medicine \& Child Neurology 2015;57(S3):34-9. [DOI: 10.1111/dmcn.12730] [PMID: 25800490]

\section{Zhao 2016}

Zhao M, Zhu P, Fujino M, Zhuang J, Guo H, Sheikh I, et al. Oxidative stress in hypoxic-ischemic encephalopathy: molecular mechanisms and therapeutic strategies. International Journal of Molecular Sciences 2016;17(12):2078. [DOI: 10.3390/ ijms17122078] [PMID: 27973415]

\section{AP P E N D I C E S}

\section{Appendix 1. Ovid MEDLINE search strategy}

Search strategy for Ovid MEDLINE(R) and Epub Ahead of Print, In-Process \& Other Non-Indexed Citations, Daily and Versions(R):

1. exp Melatonin/

2. melatonin.mp.

3. N-acetyl-5-methoxytryptamine.mp.

4. 1 or 2 or 3

5. exp Asphyxia/

6. exp Asphyxia Neonatorum/

7. exp Hypoxia-Ischemia, Brain/

8. exp Brain Ischemia/

9. exp Hypoxia/

10. exp Hypoxia, Brain/

11. exp Brain Injuries/

12. (brain injury or brain injuries).mp.

13. (neuroprotect* ${ }^{\star}$ or neuro-protect* ${ }^{\star}$ or neuro-restorative or neurorestorative).mp.

14. HIE.tw,kf.

15. encephalopath ${ }^{\star} . \mathrm{mp}$.

16. (hypoxi ${ }^{\star}$ adj2 ischaemi $\left.{ }^{\star}\right) \cdot m p$.

17. (hypoxi ${ }^{\star}$ adj2 ischemi $\left.{ }^{\star}\right) \cdot m p$. 
18. asphyxia*.mp.

19. $\left(\right.$ anoxi $^{\star}$ adj2 ischemi $\left.{ }^{\star}\right) \cdot m p$.

20. (anoxi ${ }^{\star}$ adj2 ischaemi $\left.{ }^{\star}\right) \cdot m p$.

21. or/5-20

22. exp infant, newborn/

23. (newborn* or new born or new borns or newly born or baby* or babies or premature or prematurity or preterm or pre term or low birth weight or low birthweight or VLBW or LBW or infant or infants or 'infant s' or infant's or infantile or infancy or neonat $\left.{ }^{\star}\right)$.ti,ab.

24. 22 or 23

25. randomized controlled trial.pt.

26. controlled clinical trial.pt.

27. randomized.ab.

28. placebo.ab.

29. drug therapy.fs.

30. randomly.ab.

31. trial.ab.

32. groups.ab.

33. or $/ 25-32$

34. exp animals/ not humans.sh.

35. 33 not 34

36.24 and 35

37. randomi?ed.ti,ab.

38. randomly.ti,ab.

39. trial.ti,ab.

40. groups.ti,ab.

41. ((single or doubl $l^{\star}$ or tripl ${ }^{\star}$ or treb $\left.{ }^{\star}\right)$ and (blind ${ }^{\star}$ or mask $\left.\left.{ }^{\star}\right)\right)$.ti,ab.

42. placebo*.ti,ab.

43. 37 or 38 or 39 or 40 or 41 or 42

44. 23 and 43

45. limit 44 to $y r=" 2018$-Current"

46.36 or 45

47.4 and 21 and 46

\section{Appendix 2. Cochrane Neonatal standard search strategy}

The RCT filters have been created using Cochrane's highly sensitive search strategies for identifying randomised trials (Higgins 2019). The neonatal filters were created and tested by the Cochrane Neonatal Information Specialist.

\section{CENTRAL via CRS Web:}

1. MESH DESCRIPTOR Infant, Newborn EXPLODE ALL AND CENTRAL:TARGET

2. infant or infants or infant's or "infant s" or infantile or infancy or newborn* or "new born" or "new borns" or "newly born" or neonat* or baby or babies or premature or prematures or prematurity or preterm or preterms or "pre term" or premies or "low birth weight" or "low birthweight" or VLBW or LBW or ELBW or NICU AND CENTRAL:TARGET

3. \#1 or \#2

MEDLINE via Ovid - Ovid MEDLINE(R) and Epub Ahead of Print, In-Process \& Other Non-Indexed Citations, Daily and Versions(R):

1. exp infant, newborn/

2. (newborn* or new born or new borns or newly born or baby* or babies or premature or prematurity or preterm or pre term or low birth weight or low birthweight or VLBW or LBW or infant or infants or 'infant s' or infant's or infantile or infancy or neonat*).ti,ab.

3.1 or 2

4. randomized controlled trial.pt.

5. controlled clinical trial.pt.

6. randomized.ab.

7. placebo.ab.

8. drug therapy.fs.

9. randomly.ab.

10. trial.ab.

11. groups.ab.

12. or/4-11

13. exp animals/ not humans.sh.

14. 12 not 13

15. 3 and 14

16. randomi?ed.ti,ab.

Melatonin treatment for newborns with hypoxic ischaemic encephalopathy (Protocol)

Copyright (c) 2020 The Cochrane Collaboration. Published by John Wiley \& Sons, Ltd. 
17. randomly.ti,ab.

18. trial.ti,ab.

19. groups.ti,ab.

20. ((single or doubl ${ }^{\star}$ or tripl $l^{\star}$ or treb $\left.b^{\star}\right)$ and (blind ${ }^{\star}$ or mask $\left.\left.k^{\star}\right)\right)$.ti,ab.

21. placebo*.ti,ab.

22. 16 or 17 or 18 or 19 or 20 or 21

23. 2 and 22

24. limit 23 to $y r=" 2018$-Current"

25. 15 or 24

\section{CINAHL via EBSCOhost:}

(infant or infants or infant's or infantile or infancy or newborn* or "new born" or "new borns" or "newly born" or neonat* or baby* or babies or premature or prematures or prematurity or preterm or preterms or "pre term" or premies or "low birth weight" or "low birthweight" or VLBW or LBW) AND (randomized controlled trial OR controlled clinical trial OR randomized OR randomised OR placebo OR clinical trials as topic OR randomly OR trial OR PT clinical trial)

\section{Appendix 3. Risk of bias tool}

We will use the standard methods of Cochrane and Cochrane Neonatal to assess the methodological certainty of the trials. For each trial, we will seek information regarding the method of randomisation, blinding, and reporting of all outcomes of all the infants enrolled in the trial. We will assess each criterion as being at low, high, or unclear risk of bias. Two review authors will separately assess each study. We will resolve any disagreement by discussion. We will add this information to the 'Characteristics of included studies' table. We will evaluate the following issues and enter the findings into the 'Risk of bias' table.

\section{Sequence generation (checking for possible selection bias). Was the allocation sequence adequately generated?}

For each included study, we will categorise the method used to generate the allocation sequence as:

- low risk (any truly random process, e.g. random number table; computer random number generator);

- high risk (any non-random process, e.g. odd or even date of birth; hospital or clinic record number); or

- unclear risk.

\section{Allocation concealment (checking for possible selection bias). Was allocation adequately concealed?}

For each included study, we will categorise the method used to conceal the allocation sequence as:

- low risk (e.g. telephone or central randomisation; consecutively numbered sealed opaque envelopes);

- high risk (open random allocation; unsealed or non-opaque envelopes, alternation; date of birth); or

- unclear risk

3. Blinding of participants and personnel (checking for possible performance bias). Was knowledge of the allocated intervention adequately prevented during the study?

For each included study, we will categorise the methods used to blind study participants and personnel from knowledge of which intervention a participant received. Blinding will be assessed separately for different outcomes or classes of outcomes. We will categorise the methods as:

- low risk, high risk, or unclear risk for participants; and

- low risk, high risk, or unclear risk for personnel.

4. Blinding of outcome assessment (checking for possible detection bias). Was knowledge of the allocated intervention adequately prevented at the time of outcome assessment?

For each included study, we will categorise the methods used to blind outcome assessment. Blinding will be assessed separately for different outcomes or classes of outcomes. We will categorise the methods as:

- low risk for outcome assessors;

- high risk for outcome assessors; or

- unclear risk for outcome assessors.

5. Incomplete outcome data (checking for possible attrition bias through withdrawals, dropouts, protocol deviations). Were incomplete outcome data adequately addressed? 
For each included study and for each outcome, we will describe the completeness of data including attrition and exclusions from the analysis. We will note whether attrition and exclusions were reported, the numbers included in the analysis at each stage (compared with the total randomised participants), reasons for attrition or exclusion where reported, and whether missing data were balanced across groups or were related to outcomes. Where sufficient information is reported or supplied by the trial authors, we will re-include missing data in the analyses. We will categorise the methods as:

- low risk (less than $20 \%$ missing data);

- high risk (20\% missing data or more); or

- unclear risk.

\section{Selective reporting bias. Are reports of the study free of suggestion of selective outcome reporting?}

For each included study, we will describe how we investigated the possibility of selective outcome reporting bias and what we found. For studies in which study protocols were published in advance, we will compare prespecified outcomes versus outcomes eventually reported in the published results. If the study protocol was not published in advance, we will contact study authors to gain access to the study protocol. We will assess the methods as:

- low risk (where it is clear that all of the study's prespecified outcomes and all expected outcomes of interest to the review have been reported);

- high risk (where not all the study's prespecified outcomes have been reported; one or more reported primary outcomes were not prespecified outcomes of interest and are reported incompletely and so cannot be used; or the study fails to include results of a key outcome that would have been expected to have been reported); or

- unclear risk.

\section{Other sources of bias. Was the study apparently free of other problems that could put it at a high risk of bias?}

For each included study, we will describe any important concerns we had about other possible sources of bias (for example, whether there was a potential source of bias related to the specific study design or whether the trial was stopped early due to some data-dependent process). We will assess whether each study was free of other problems that could put it at risk of bias as:

- low risk;

- high risk; or

- unclear risk.

If needed, we plan to explore the impact of the level of bias through undertaking sensitivity analyses.

\section{H I S T O R Y}

Protocol first published: Issue 10, 2020

\section{CONTRIBUTIONS OF AUTHORS}

All authors contributed to writing and reviewing the protocol.

\section{DECLARATIONS OF INTEREST}

TH: under the supervision of Professor Eleanor Molloy, Tim Hurley is conducting research on the ex-vivo effects of melatonin treatment on inflammation in newborns with NE. He is a PhD student funded by the Health Research Board of Ireland (Sources of support).

MO'D has no interests to declare.

SA has no interests to declare.

HA has conducted and published a randomised controlled pilot study of melatonin for neuroprotection in newborns with perinatal asphyxia.

NR has conducted pre-clinical trials of melatonin for neuroprotection in animal model studies of neonatal encephalopathy. In 2016 her institution licensed an orphan drug for melatonin for treatment of birth asphyxia to Chiesi Pharmaceuticals. Her institution (University College London) received a research grant from Chiesi Pharmaceuticals for a pre-clinical study involving the use of their melatonin formulation.

EM's institution receives a grant from the National Children's Research Centre Health Research Board Ireland. Professor Molloy is Associate Editor in Chief for the journal Pediatric Research. Her institution receives support from Pediatric Research for Professor Molloy to travel to ESPR and PAS. She is a National Children's Research Centre board member but receives no payment for this. 


\section{SOURCES OF SUPPORT}

\section{Internal sources}

- Health Research Board, Ireland

Funding for PhD Student, Tim Hurley

\section{External sources}

- Vermont Oxford Network, USA

Cochrane Neonatal Reviews are produced with support from Vermont Oxford Network, a worldwide collaboration of health professionals dedicated to providing evidence-based care of the highest quality for newborn infants and their families 